\title{
Lumbosacral Sagittal Alignment in Association to Intervertebral Disc Diseases
}

\author{
Zohreh Habibi $^{1,2}$, Farid Maleki ${ }^{1}$, Ali Tayebi Meybodi ${ }^{3}$, Ali Mahdavi ${ }^{4}$, Hooshang Saberi ${ }^{1,3}$ \\ ${ }^{1}$ Brain and Spinal Cord Injuries Repair Research Center (BASIR), Tehran University of Medical Sciences, Tehran, Iran \\ ${ }^{2}$ Department of Neurosurgery, Children's Medical Center Hospital, Tehran University of Medical Sciences, Tehran, Iran \\ ${ }^{3}$ Department of Neurosurgery, Imam Khomeini Hospital, Tehran University of Medical Sciences, Tehran, Iran \\ ${ }^{4}$ Department of Radiology, Sina Hospital, Tehran University of Medical Sciences, Tehran, Iran
}

\begin{abstract}
Study Design: A cross-sectional case-control study was designed to compare the sagittal alignment of lumbosacral regions in two groups of patients suffering from low back pain, one with intervertebral disc pathologies and one without.

Purpose: To evaluate the correlation between lumbosacral sagittal alignment and disc degeneration.

Overview of Literature: Changes in lumbar lordosis and pelvic parameters in degenerative disc lesions have been assessed in few studies. Overall, patients with discopathy were shown to have lower lumbar lordosis and more vertical sacral profiles.

Methods: From patients with intractable low back pain undergoing lumbosacral magnetic resonance imaging, 50 subjects with disc degeneration and 50 controls with normal scans were consecutively enrolled. A method was defined with anterior tangent-lines going through anterior bodies of L1 and S1 to measure global lumbosacral angle, incorporating both lumbar lordosis and sacral slope. Global lumbosacral angle using the proposed method and lumbar lordosis using Cobb's method were measured in both groups.

Results: Lumbar lordosis based on Cobb's method was lower in group with discopathy $\left(20^{\circ}-67^{\circ}\right.$; mean, $\left.40.48^{\circ} \pm 9.89^{\circ}\right)$ than control group $\left(30^{\circ}-62^{\circ}\right.$; mean, $\left.44.96^{\circ} \pm 7.68^{\circ}\right)$, although it was not statistically significant. The proposed global lumbosacral angle in subject group $\left(53^{\circ}-103^{\circ}\right.$; mean, $\left.76.5^{\circ} \pm 11.018^{\circ}\right)$ was less than control group $\left(52^{\circ}-101^{\circ}\right.$; mean, $\left.80.18^{\circ} \pm 9.95^{\circ}\right)$, with the difference being statistically significant $(p=0.002)$.

Conclusions: Patients with intervertebral disc lesions seem to have more straightened lumbosacral profiles, but it has not been proven which comes first: disc degeneration or changes in sagittal alignment. Finding an answer to this dilemma demands more comprehensive long-term prospective studies.
\end{abstract}

Keywords: Lumbosacral Region; Sagittal; Global lumbosacral angle; Intervertebral disc degeneration; Low back pain

\section{Introduction}

The functional and clinical importance of lumbar lordosis is being recognized increasingly [1,2]. Loss of normal lordotic alignment may induce pathologic changes in the spine from load bearing, and accelerate degeneration of the functional motion units [3]. Consequently, the analysis of sagittal balance recently seems to be essential in the management of lumbar degenerative pathologies [4]. Clinicians have described different ways to evaluate lumbar lordosis, with the Cobb's method being the most prevalent. Investigations to compare different methods,

Received Dec 23, 2013; Revised Feb 20, 2014; Accepted Feb 20, 2014

Corresponding author: Zohreh Habibi

Department of Neurosurgery, Children's medical Center Hospital, Tehran University of Medical Sciences,

Gharib Street, Tehran, Iran, 1419733141

Tel: +98-2161192634-5, Fax:+982161192774, E-mail: zohreh_h56@yahoo.com 
namely Centroid, Trall, Harrison posterior tangent lines, and Cobb's, showed that all of these have high reliability and low absolute inter-observer differences [5]. Currently, modern software modeling and characterizing the shape of lumbar spine has been introduced [6].

Abnormal spinal sagittal alignment can cause persistent low back pain (LBP) and the association of acute LBP with hyperlordosis, and the relationship of chronic LBP with hypolordosis have been demonstrated $[7,8]$.

In contrast to numerous discussions regarding the relationship between lordosis and LBP, only a few controlled studies have assessed the correlation of lumbar sagittal alignment to degenerative disc disease (DDD) or disc herniation (DH) [7]. Spino-pelvic morphology and sacral alignment received even less attention in this regard $[4,9,10]$.

In current study, we defined a new method for measuring the global lumbosacral angle incorporating both lumbar lordosis and sacral slope. The objective of the study was to assess the correlation of lumbosacral sagittal alignment (using this proposed technique and Cobb's method) to disc degeneration, in patients suffering from LBP.

\section{Materials and Methods}

\section{Study design}

A Cross-sectional case-control study was designed to compare the sagittal alignment of lumbosacral area in two groups of patients suffering from LBP: one with intervertebral disc pathologies and one without.

\section{Subjects}

Among the patients who visited an outpatient back pain clinic, those with recent LBP (developed or exacerbated within last two to eight weeks) without nerve root irritation symptoms were initially planned for treatment with nonsteroidal anti-inflammatory drug, enough rest, physical therapy, and back school exercises. Those who did not improved after four weeks of such treatments and whose pain severity based on visual analog scale (VAS) was reported to be between 5 and 7, were invited to undergo lumbosacral magnetic resonance imaging (MRI). The patients with VAS of more than 8 were excluded from the program and were referred to instant evaluation and treatment. From 724 patients who were designated for lumbosacral MRI, only 686 accepted to undergo MRI, among whom 50 subjects ( 25 males and 25 females) with disc degeneration were consecutively enrolled according to inclusion/exclusion criteria. Subsequently, among the same population, 50 controls with LBP but normal MRI were matched for age, sex, and body mass index (BMI). Informed consent was obtained before enrollment, and the study was approved by the Institutional Medical Research and Ethics Committee.

Patients were excluded from the study if they had conditions as follows: radicular pains and paresthesia; history of rheumatologic diseases; apparent spinal deformity shown in clinical exam; previous spinal surgery, including simple lumbar discectomy; spinal mass lesions; old fractures; spondylolysis and spondylolisthesis; scoliosis; and overt bony anomalies such as spina bifida, block vertebra, lumbarization, and sacralization; and age of less than 17 years or more than 65 years.

\section{Imaging parameters}

MRI was performed with $1.5 \mathrm{~T}$ magnetic field unit using a standardized scan protocol for all subjects and controls, with participants lying in supine position with extended legs. Sagittal T1 weighted (repetition time [TR]/echo time [TE]: 540-11 ms, spacing: $3.3 \mathrm{~mm}$, slice thickness: $3 \mathrm{~mm}$ ), sagittal T2 weighted (TR/TE: $2800-79 \mathrm{~ms}$, spacing: $3.3 \mathrm{~mm}$, slice thickness: $3 \mathrm{~mm}$ ), and axial T2 weighted (TR/TE: $4680-10 \mathrm{~ms}$, spacing: $4.4 \mathrm{~mm}$, slice thickness: $4 \mathrm{~mm}$ ) sequences were used in the imaging protocol. T1and T2-weighted images were used to assess morphologic parameters and pathological discogenic changes, respectively.

\section{Measurements of lumbar lordosis and global lumbo- sacral angle}

Cobb's method: Lumbar lordotic angle was measured between the lines drawn along inferior endplate of T12 and superior end plate of S1 (Fig. 1A).

Proposed method: The global lumbosacral angle (LS angle) was defined as an angle between the tangent lines passing through the anterior bodies of L1 and S1 (Fig. 1B). Avoiding pointed edges/ marginal osteophytes, the tangent lines were passed through the smooth middle portion of the anterior walls.

The angle between the anterior and posterior surfaces 
of the first sacral vertebral body (S1 angle) was described to assess 'funneling profile' of the S1 body (Fig. 1C).

The measurements were performed twice using AutoCAD software (version 2012) by single author (F.M.) and then rechecked using random selection by another author (Z.H.). The inter-observer and intra-observer validity was checked, and an average of three records of each measurement were used for statistical analysis to minimize
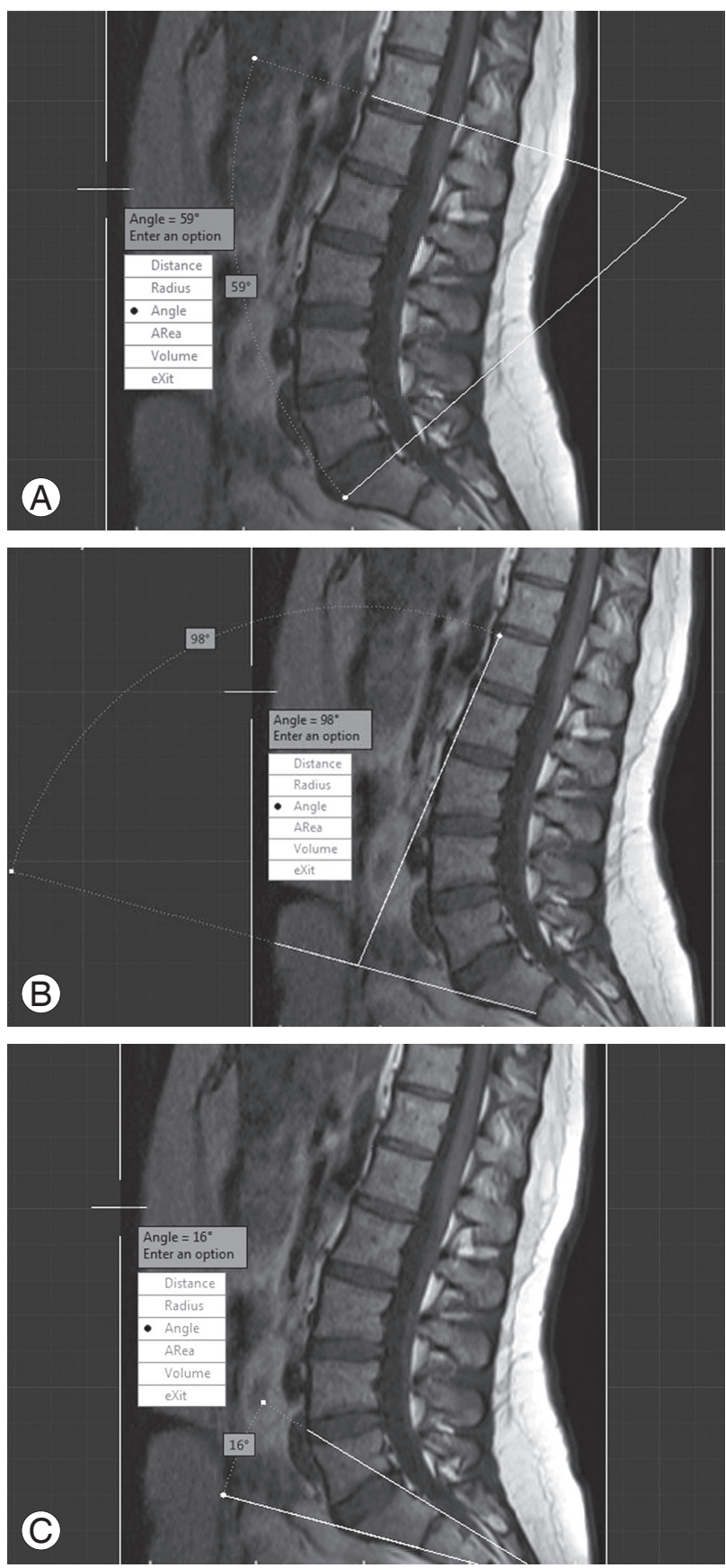

Fig. 1. (A) Total lumbar lordosis using Cobb's method. (B) Global lumbosacral angle using proposed method. (C) S1 funneling angle. random error.

\section{Evaluation of discal degenerative features}

The presence and degree of intervertebral disc pathologies was assessed on T2-weighted images of midsagittal and parasagittal planes and axial cuts through disc spaces. According to the assessment, discal degenerative features were classified into one of the four possible categories including Pfirrmann grade III to V changes [11], disc bulging plus Pfirrmann grade III to $\mathrm{V}$ changes, disc protrusion, and disc Extrusion, which were numbered as classes 1, 2, 3, and 4, respectively.

Pfirrmann grades I and II were considered normal (Table 1).

\section{Statistical analysis}

For each variable, mean and standard deviation values were calculated and the differences between the subject and control groups were examined using independent t-test. Statistical analyses were carried out by SPSS ver. 16.0 (SPSS Inc., Chicago, IL, USA) and the level of significance was defined by $p$-value less than 0.05 . Moreover, intra-rater and inter-rater reliability of the new angle was determined via intraclass correlation coefficient (ICC), 95\% confidence intervals (CI), and standard error. The following categories for expressing levels of reliability were used: 0.40 , poor reliability; 0.40 to 0.75 , fair to good reliability; and 0.90 , perfect reliability.

\section{Results}

There was a population of 50 subjects ( 25 males and 25 females) and 50 controls ( 25 males and 25 females) in this study. The mean age was $36.71 \pm 10.328$ years (range, $17-58$ years).

The mean, standard deviation, maximum values, and minimum values for lumbar lordosis using Cobb's method and those values for proposed global lumbosacral angle in two groups are shown in Table 2.

\section{Intra-observer and inter-observer reliability of the proposed anterior tangent line method}

For the first step, the inter-observer and intra-observer reliability of the new method was examined by ICC 
Table 1. Pfirrmann grading system for disc degeneration

\begin{tabular}{lllll} 
Grade & Disc height & $\begin{array}{l}\text { Annulus-nucleus } \\
\text { distinction }\end{array}$ & Feature & Signal intensity \\
\hline I & Normal & Clear & $\begin{array}{l}\text { Bright } \\
\text { white internal structure }\end{array}$ & Hyperintense \\
II & Normal & Clear & Inhomogeneous white internal structure & Hyperintense \\
III & $\begin{array}{l}\text { Normal to slightly } \\
\text { decreased }\end{array}$ & Not entirely clear & Grey disc with inhomogeneous internal & Intermediate \\
IV & $\begin{array}{l}\text { Normal to moderately } \\
\text { decreased }\end{array}$ & Not clear & Greycture & internal structure \\
V & Severely decreased & Not clear & Black disc with inhomogeneous internal & Hypointense \\
\hline
\end{tabular}

Table 2. Lumbar lordosis with Cobb's method and proposed global lumbosacral angle in patients suffering from low back pain, with or without discopathy

\begin{tabular}{|c|c|c|c|c|c|c|c|c|}
\hline \multirow{2}{*}{ Angle } & \multicolumn{4}{|c|}{ Patients with discopathy } & \multicolumn{4}{|c|}{ Patients without discopathy } \\
\hline & Mean & SD & Min & $\operatorname{Max}$ & Mean & SD & Min & $\operatorname{Max}$ \\
\hline Lumbar lordosis & 40.48 & 9.896 & 20 & 67 & 44.96 & 7.688 & 30 & 62 \\
\hline Global lumbosacral angle & 76.58 & 11.018 & 53 & 103 & 80.18 & 9.956 & 52 & 101 \\
\hline
\end{tabular}

SD, Standard deviation; Min, minimum value; Max, maximum value.

(model 2 and 3). There was a perfect agreement between the two series of values obtained by the first rater (F.M.) to assess intra-observer reliability (ICC, $0.940 ; 95 \%$ CI, 0.88-0.97). Likewise, the average of the two measurements from the primary rater (F.M.) was compared with the single measurement from the secondary rater (Z.H.) to calculate inter-observer reliability, and it displayed a perfect agreement (ICC, 0.920; 95\% CI, 0.84-0.96).

\section{Correlation between disc degeneration and lumbar/ lumbosacral sagittal alignment}

Lumbar lordosis based on two-line Cobb's method was lower in subject group $\left(20^{\circ}\right.$ to $67^{\circ}$; mean, $\left.40.48^{\circ} \pm 9.89^{\circ}\right)$ compared to control group $\left(30^{\circ}\right.$ to $62^{\circ}$; mean, $44.96^{\circ} \pm$ $\left.7.68^{\circ}\right)$, although it is not statistically significant. The proposed global lumbosacral angle was between $53^{\circ}$ and $103^{\circ}\left(76.5^{\circ} \pm 11.018^{\circ}\right)$ in subject group which was again less than the values in control group $\left(52^{\circ}-101^{\circ}\right.$; mean, $\left.80.18^{\circ} \pm 9.95^{\circ}\right)$, with the difference being statistically significant $(p=0.002)$. The mean $\mathrm{S} 1$ angle was $18.7^{\circ}$ in subject group and it was $19.96^{\circ}$ in control groups, which implies that the patient with disc degeneration had less sharpness of S1. Even so, the difference was not significant.

None of the measured angles showed significant differences in the subgroups of degenerative lesions (i.e., Pfirrmann III-V, bulging, protrusion, and extrusion).

\section{Discussion}

LBP is one of the most frequent causes of medical visits all throughout the world. The prevalence of LBP in adult population has been recorded as high as $60 \%$ to $90 \%$ [9]. The association between changes in lumbar lordotic alignment and LBP is well described in medical literatures $[12,13]$. However, previous studies mainly assessed lumbar lordosis and pelvic parameters in LBP or spondylolisthesis, while few of them concerned these issues in disc degeneration $[4,7]$. Considering the fact that discogenic pain is one of the main causes of LBP [14], evaluating its associated factors such as alteration in lumbar lordosis and sacral parameters seems invaluable.

Lumbar lordosis is formed by the wedging of the lumbar vertebral bodies and intervertebral discs [15]. Overall, the degree of lumbar lordosis decreases with advanced age [16]. The changes in sagittal alignment may lead to changes in the lumbar spine kinematics, which will sub- 
sequently influence on load bearing and the occurrence of disc degeneration [3]. This biomechanical finding is not merely restricted to lumbar vertebrae. The changes in sagittal alignment of cervical spine may also cause changes in maximum load and accelerate degeneration [17].

In current study, we analyzed lumbar/lumbosacral alignment for potential associations to intervertebral disc pathologies. All measurements were performed on supine MRI, as done in some of the other analogous studies [2,9]. Standing MRI which was the alternative method to standing X-ray was not available in our center, so we chose the method of supine MRI. The logic behind performing MRI instead of standing X-ray was to avoid unnecessary irradiation to the population most of whom were in their reproductive age (17 to 65 years). Moreover, recent investigations revealed that lumbar lordosis measured on horizontal MRI in supine position with straight legs was comparable to that measured on vertical standing images [18], and sagittal balance obtained in supine MRI is reliable for investigational studies [2].

The rationale for comparing two groups of patients with LBP, with and without disc degeneration, is that LBP can influence sagittal alignment by itself. Sciatica may also change sagittal alignment. Consequently, subject and control groups were both selected among the patients suffering from LBP of the same intensity (VAS 5 to 7) without sciatica, in order to eliminate the confounding effects of such factors and to compare the sagittal alignment in two groups with identical conditions.

\section{Lumbar lordosis versus global lumbosacral angle in association with disc degeneration}

In this study, the lumbar lordosis obtained by two-line Cobb's method was lower in the subjects with LBP and disc degeneration/herniation compared to those with LBP without discopathy. Although statistically insignificant, this finding is in concordance with the results of few similar studies. Barrey et al. [4] have shown lumbar lordosis to be significantly lower in patients with $\mathrm{DH}$ or DDD. Endo et al. [7] demonstrated that the lordotic angle between the top margins of L1 and S1 was smaller in patients with $\mathrm{DH}$, but returned to almost the same level as the control group six month after surgical treatment. In a study evaluating the degree of disc degeneration, Ergun et al. [9] found that the degree of degeneration increased parallel to the decrease in the lumbar lordotic angles.
However, this issue is still a matter of controversy; for instance, Kalichman et al. [2] and Lebkowski et al. [19] did not find significant correlation between diminished lordosis and lumbar degenerative disc lesions.

Summating both sacral sharpness and lumbar lordosis as global lumbosacral angle, a statistically significant association to lumbar degenerative disc pathologies was found in our series. Furthermore, in subject group the body of S1 exhibited less funneling in shape, which is comparable with other investigations focusing on more vertical sacrum in patients with lumbosacral discopathy $[7,10]$. Nevertheless, S1 angle was not significantly different between the subjects and the controls; besides, the difference of lumbar lordosis between the two groups was not significant in our study. So, it can be proposed that the sum of whole regional curvatures may have more rigorous associations to disc degeneration, compared to either lumbar lordosis or sacral sharpness. Consequently, it is better to use global lumbosacral curve rather than lumbar lordosis for more accurate assessment of sagittal alignment and its potential correlation to degenerative diseases. Indeed, it has now been just established that to analyze sagittal balance of a subject, it is fundamental to include the spatial position of the pelvis and its shape [4]. In the last decade, a few studies have evaluated the sacrum shape and the slope in lumbar intervertebral disc diseases. In a study performed in 2002, lower sacral slope and more straightened lumbar curvature were found in patients with DH [10]. In 2007, Barrey et al. [4] showed that the patients with disc lesions were characterized by a low or normal 'pelvic incidence', a morphological parameter incorporating sacral slope and pelvic tilt. In a study by Endo et al. [7], the patients with DH exhibited more vertical sacrum than control groups; and Ergun et al. [9] demonstrated that sacral kyphosis was significantly diminished in those with disc degeneration.

The current study introduces a new parameter to quantify the global lumbosacral curve and confirms that the lumbosacral sagittal profile is straighter in lumbar discopathy. The function of natural inclination of lumbosacral joint and lumbar lordosis is to protect against degenerative damages. As the lumbosacral column becomes straighter, intervertebral discs may more easily be subjected to degeneration [9]. The potential cause in this situation is the gravitational force increasing the compressive component over the area $[10,20,21]$. 


\section{Sagittal misalignment; cause or effect of degenerative processes}

As previously mentioned, some studies have found that patients with DDD/DH may show loss of lumbar lordosis. It is supposed that the loss of lordosis in discopathy is not only structural due to segmental loss of disc height, but also postural due to the analgesic posture of the patient to avoid posterior discal hyperpressure [4]. In the present study, both subject and control groups were included if the VAS for pain was between 5 and 7, and since the pain scale was roughly similar, the postural component would not be much different between the two groups. Also, patients with DH may sometimes present with a forwardbending posture while walking, because the sagittal balance might have been affected by sciatic stimulation and tonic contraction of the surrounding lumbopelvic muscles [7]. Endo et al. [7] proposed that through the pain relief for 6 months after removing extruded disc, the mal-alignment recovered to almost the same level as the control group. Decreased lumbar lordosis may also be found in lumbar degenerative diseases other than disc extrusion with radiculopathy. For instance, patients with canal stenosis and claudication were shown to have even smaller lordotic angle than those with nerve root symptoms [22]. In the current study, subjects with diminished lumbosacral curve had pure LBP without nerve irritation. Consequently, changes in sagittal alignment can occur in patients with LBP and disc degeneration, regardless of the presence or absence of sciatica. Hence, the nerve root irritation is not a mere requisite to create spinal misalignment. This sagittal misalignment would even be the cause rather than the result of disc degeneration, or at least, the two phenomena may have some reciprocal effects on each other. Indeed, a diminished lumbar lordosis combined with a more vertical sacrum means a more straightened lumbosacral profile, which in turn will increase the compressive gradient over the area that may accelerate degeneration and consequently exacerbate the misalignment. So, a vicious cycle is formed which is analogous to the classic case questioning what comes first, the chicken or the egg. A more probable conclusion would be that the disc degeneration is the cause of changes in lumbosacral curvature, not the other way around. To solve this dilemma, a longitudinal prospective assessment of healthy samples is required to monitor the evolution of disc degeneration and potential changes in lumbosacral alignment.

\section{Shortcomings and needs for further studies}

There are several limitations in the current study. The sample size was relatively small and longitudinal followups were not performed to assess the influences of lumbosacral profile on disc degeneration, or vice versa. This was due to the cross-sectional design of this study. Moreover, all measurements were performed in supine position. Although both groups had identical positions during imaging and some former studies had demonstrated that sagittal alignment measured in supine position is reliable for investigational analyses $[2,9,18,23]$, standing position may be a standard one in general. Other supplementary parameters affecting sagittal balance, such as sacral slope and pelvic incidence, were not obtained in this study due to lack of standing plain film (although S1 funneling profile was introduced to estimate sacral sharpness). Addressing such parameters would allow carrying out a multivariate analysis and enhancing the strength of the evidence supporting the conclusion. Nevertheless, since the two groups were matched as closely as possible, acceptable conclusions could be drawn from the present study; although the power of such conclusions is not comparable to a multivariate analysis. This conclusion is applicable to "global lumbosacral angle" only, and not to the whole sagittal balance.

The results of this study need to be replicated in larger sample, preferably by using longitudinal design and by incorporating all possible variables.

\section{Conclusions}

The results of this study show that the global lumbosacral angle measured in supine position with straight legs is lower in patients with lumbar disc degeneration and/or herniation. However, the dilemma of which coming first, sagittal imbalance or disc degeneration remains to be answered. Definite response to this question demands a longitudinal prospective evaluation of healthy samples to monitor the evolution of disc degeneration and changes in lumbosacral alignment.

\section{Conflict of Interest}

No potential conflict of interest relevant to this article was reported. 


\section{References}

1. Jang JS, Lee SH, Min JH, Maeng DH. Influence of lumbar lordosis restoration on thoracic curve and sagittal position in lumbar degenerative kyphosis patients. Spine (Phila Pa 1976) 2009;34:280-4.

2. Kalichman L, Li L, Hunter DJ, Been E. Association between computed tomography-evaluated lumbar lordosis and features of spinal degeneration, evaluated in supine position. Spine J 2011;11:308-15.

3. Keorochana G, Taghavi CE, Lee KB, et al. Effect of sagittal alignment on kinematic changes and degree of disc degeneration in the lumbar spine: an analysis using positional MRI. Spine (Phila Pa 1976) 2011;36: 893-8

4. Barrey C, Jund J, Noseda O, Roussouly P. Sagittal balance of the pelvis-spine complex and lumbar degenerative diseases. A comparative study about 85 cases. Eur Spine J 2007;16:1459-67.

5. Harrison DE, Harrison DD, Cailliet R, Janik TJ, Holland B. Radiographic analysis of lumbar lordosis: centroid, Cobb, TRALL, and Harrison posterior tangent methods. Spine (Phila Pa 1976) 2001;26:E235-42.

6. Meakin JR, Gregory JS, Smith FW, Gilbert FJ, Aspden RM. Characterizing the shape of the lumbar spine using an active shape model: reliability and precision of the method. Spine (Phila Pa 1976) 2008;33:807-13.

7. Endo K, Suzuki H, Tanaka H, Kang Y, Yamamoto K. Sagittal spinal alignment in patients with lumbar disc herniation. Eur Spine J 2010;19:435-8.

8. Harrison DD, Cailliet R, Janik TJ, Troyanovich SJ, Harrison DE, Holland B. Elliptical modeling of the sagittal lumbar lordosis and segmental rotation angles as a method to discriminate between normal and low back pain subjects. J Spinal Disord 1998;11:430-9.

9. Ergun T, Lakadamyali H, Sahin MS. The relation between sagittal morphology of the lumbosacral spine and the degree of lumbar intervertebral disc degeneration. Acta Orthop Traumatol Turc 2010;44:293-9.

10. Rajnics P, Templier A, Skalli W, Lavaste F, Illes T. The importance of spinopelvic parameters in patients with lumbar disc lesions. Int Orthop 2002;26:104-8.

11. Pfirrmann CW, Metzdorf A, Zanetti M, Hodler J, Boos N. Magnetic resonance classification of lumbar intervertebral disc degeneration. Spine (Phila $\mathrm{Pa}$ 1976) 2001;26:1873-8.

12. Evcik D, Yucel A. Lumbar lordosis in acute and chronic low back pain patients. Rheumatol Int 2003; 23:163-5.

13. Youdas JW, Garrett TR, Egan KS, Therneau TM. Lumbar lordosis and pelvic inclination in adults with chronic low back pain. Phys Ther 2000;80:261-75.

14. Salminen JJ, Erkintalo MO, Pentti J, Oksanen A, Kormano MJ. Recurrent low back pain and early disc degeneration in the young. Spine (Phila Pa 1976) 1999; 24:1316-21.

15. Vialle R, Levassor N, Rillardon L, Templier A, Skalli W, Guigui P. Radiographic analysis of the sagittal alignment and balance of the spine in asymptomatic subjects. J Bone Joint Surg Am 2005;87:260-7.

16. Gelb DE, Lenke LG, Bridwell KH, Blanke K, McEnery KW. An analysis of sagittal spinal alignment in 100 asymptomatic middle and older aged volunteers. Spine (Phila Pa 1976) 1995;20:1351-8.

17. Miyazaki M, Hymanson HJ, Morishita Y, et al. Kinematic analysis of the relationship between sagittal alignment and disc degeneration in the cervical spine. Spine (Phila Pa 1976) 2008;33:E870-6.

18. Andreasen ML, Langhoff L, Jensen TS, Albert HB. Reproduction of the lumbar lordosis: a comparison of standing radiographs versus supine magnetic resonance imaging obtained with straightened lower extremities. J Manipulative Physiol Ther 2007;30:26-30.

19. Lebkowski WJ, Lebkowska U, Niedzwiecka M, Dzieciol J. The radiological symptoms of lumbar disc herniation and degenerative changes of the lumbar intervertebral discs. Med Sci Monit 2004;10 Suppl 3:112-4.

20. O'Connell GD, Johannessen W, Vresilovic EJ, Elliott DM. Human internal disc strains in axial compression measured noninvasively using magnetic resonance imaging. Spine (Phila Pa 1976) 2007;32:2860-8.

21. O'Connell GD, Vresilovic EJ, Elliott DM. Human intervertebral disc internal strain in compression: the effect of disc region, loading position, and degeneration. J Orthop Res 2011;29:547-55.

22. Suzuki H, Endo K, Kobayashi H, Tanaka H, Yamamoto $\mathrm{K}$. Total sagittal spinal alignment in patients with lumbar canal stenosis accompanied by intermittent claudication. Spine (Phila Pa 1976) 2010;35:E344-6.

23. Abdel MP, Bodemer WS, Anderson PA. Supine thoracolumbar sagittal spine alignment: comparing computerized tomography and plain radiographs. Spine (Phila Pa 1976) 2012;37:340-5. 